\title{
Kinder und Jugendliche fördern und fordern: E.ON Avacon sponsert "Kinder gestalten ihre Stadt"
}

K

inder sind kreativ. Genau diese Kreativität fördern Projekte wie „Kinder gestalten ihre Stadt“, das E.ON Avacon über den Verein „Kunst im Bahnhof“ finanziell unterstützt hat. „Kinder gestalten ihre Stadt“ nimmt diesen Gedanken an einem ganz konkreten Beispiel auf und lässt Kinder ein Umfeld gestalten, in dem sie sich wohl und zu Hause fühlen. Damit kanalisiert das Projekt die Kreativität in konkret umsetzbare Bahnen und zeigt, wie Ideen von Kindern auch unser Leben heute positiver, lebensfroher machen können.

Denn auch in Zukunft wird Kreativität unsere Gesellschaft, unser Leben wesentlich beeinflussen. Fördern und fordern wir unsere Kinder in diesem Sinne, dann legen Projekte wie dieses heute schon ein wichtiges Fundament für ihre Zukunft.

Als Energiedienstleister für weite Teile Niedersachsens und Sachsen-Anhalts fühlen wir uns den Regionen, in denen wir arbeiten, und den Menschen, die dort leben, verbunden und verpflichtet. E.ON Avacon unterstützt deshalb in vielfältiger Form wissenschaftliche, kulturelle und sportliche Projekte und übernimmt damit soziale Verantwortung. Einen ganz wesentlichen Schwerpunkt bei der Auswahl der Sponsoringprojekte setzen wir dabei auf die Nachwuchsförderung.

Deshalb freuen wir uns sehr, ein solches zukunftsweisendes und innovatives Projekt zu unterstützen. Und wir hoffen, dass durch die Ergebnisse jedem bewusst wird: Kinder sind unsere Zukunft und Kinder können unsere Zukunft bereits jetzt ganz konkret mitgestalten.

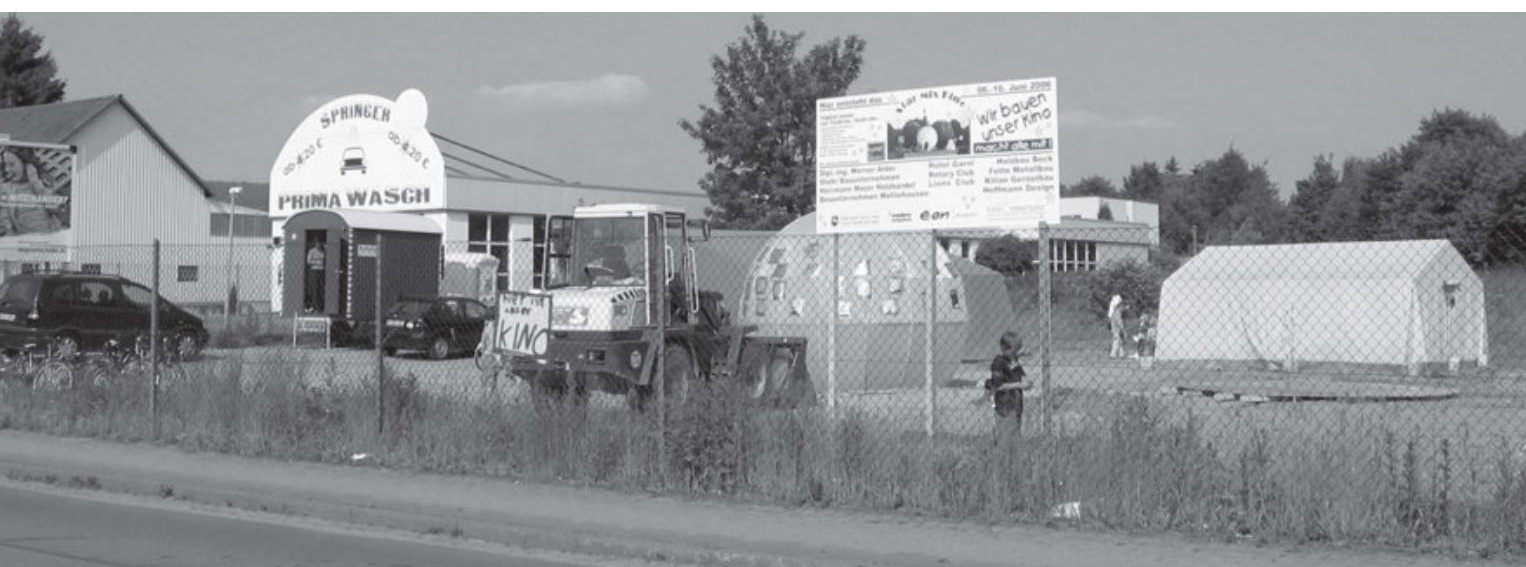




\section{Kinder als Filmstars - hinter der Kamera}

Kino-Projektwoche des Vereins „Kunst im Bahnhof" geht mit einem Abschlussfest zu Ende

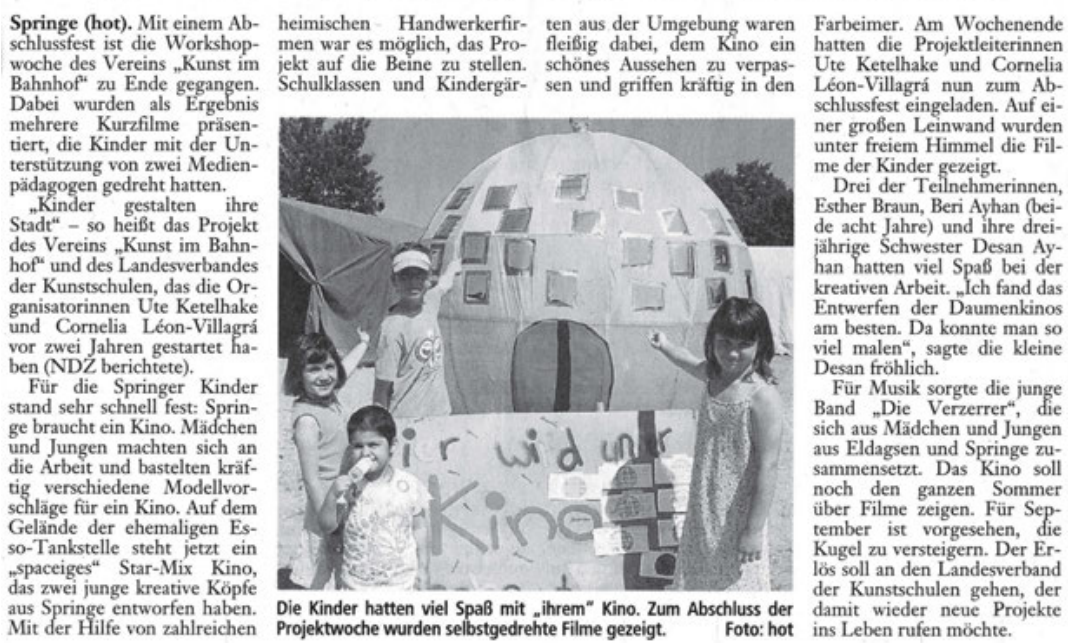

Neue Deister Zeitung, 13.06.2006

\section{Heute keine Hausaufgaben, morgen Schauspieler}

Sommerkino des Vereins „Kunst im Bahnhof“ eröffnet / Bis Sonnabend täglich um 17 Uhr kostenlose Filmnachmittage

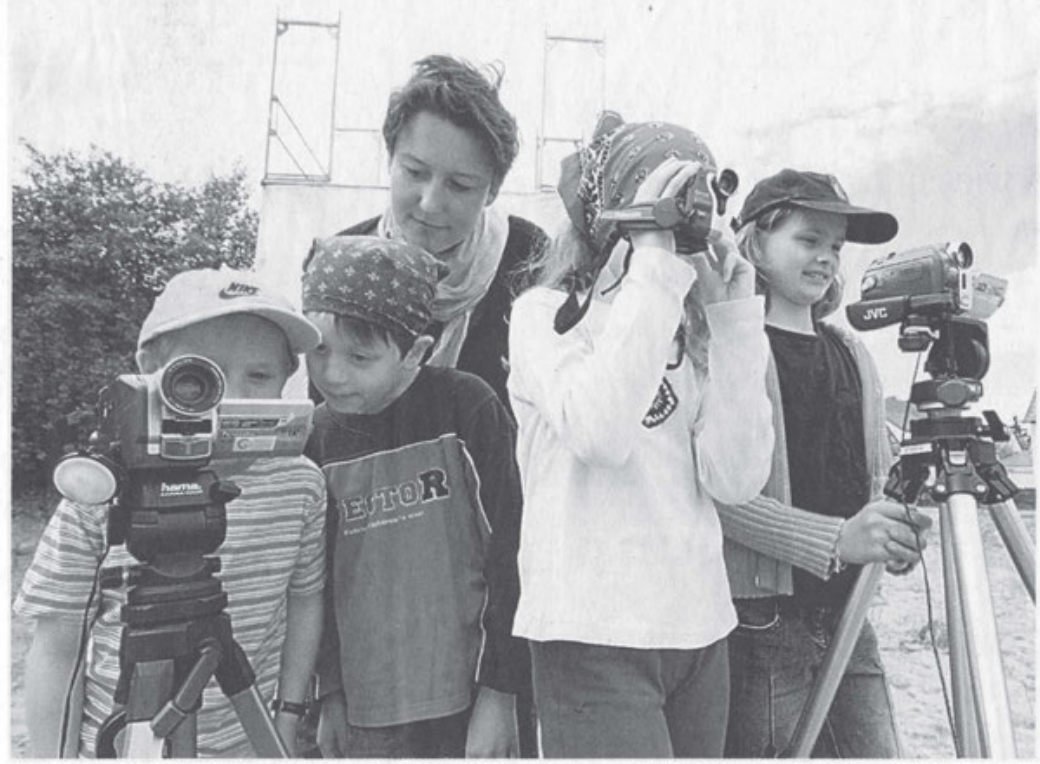

Die Kamerakinder der $2 \mathrm{~b}$ hatten gestern den vollen Durchblick: Justin, Robert. Melanie und Johanna (v. I.) wurden von der Kulturpădagogin Laura van Joolen ins Filmgeschäft eingewiesen. Im Hintergrund ist die große Leinwand, auf der abends Kinostreifen laufen sollen, zu sehen.
Springe (mari). „Umsonst und draußen" - Der Verein "Kunst im Bahnhof" hat ges. tern das erste Springer Sommerkino auf dem Gelinnde der merkino auf dem Gelände der
ehemaligen Esso-Tankstelle eröffnet. Heute um $17 \mathrm{Uhr}$ zeigen die Organisatoren den Streifen Hilfe, ich bin ein Junge", dazu gibt es mehrere Film- und Video-Workshops.

Die 2b der Grundschule Hinter der Burg war die erste, die die Filmlandschaft betreten durfte. Morgen kommen drei Kindergärten. „Leider war das Interesse der Schulen nur mäbig", ist Mitorganisatorin Cornelia León-Villagra enträuscht. Schade ist das vor enttäuscht. Schade ist das vor
allem, weil die beiden Meallem, weil die beiden Medienpädagogen Renzo Solór-
zano und Laura van Joolen jetzt zwangsläufig Leerlauf haben.

Am Nachmittagsprogramm Am Nachmittagsprogramm
andert das nichts: Heute, morgen und übermorgen bietet der Verein von 1 है bis 18 Uhr Filme und Spiele an. Wer den Kinofilm um 17 Uhr sehen möchte, sollte sich ein Kissen mitbringen.

Die $2 \mathrm{~b}$ kam bereits zur gestrigen Fortsetzung wieder: lhre Lehrerin Sabine KünneBornemann verzichtete extra auf die Hausaufgaben, "damit die Kinder nachmittags die Früchte ernten können, die sie vormittags gesät haben". 